\title{
RESEARCH
}

Open Access

\section{Telovelar approach versus transvermian approach in management of fourth ventricular tumors}

\author{
Khaled Saeed Ebrahim ${ }^{*}$ (D) and Ahmed Faisal Toubar
}

\begin{abstract}
Background: The relationships of the fourth ventricle to the cerebellar surfaces and the fissures through which the fourth ventricle is approached surgically are among the most complex in the brain. The area in and around the fourth ventricle may be affected by a variety of neoplasms. Some are unique to the fourth ventricle; others are also found in different locations. The two most common surgical approaches to the fourth ventricle are the transvermian and the telovelar approaches.

The aim of this study: The aim of this study is to compare the results of telovelar and transvermian approaches in the management of fourth ventricular tumors with regard to clinical outcome and extent of tumor excision.

Patients and methods: This study includes 40 patients presented at Ain Shams University Hospital and Nasser Institute with the diagnosis of fourth ventriclular tumors in a 4-year period between 2011 and 2015. Surgical treatment was done in all cases in form of (1) a CSF diversion for hydrocephalic patients through insertion of V-P shunt or EVD. (2) Completely randomly choosing an approach, 20 patients were operated through the transvermian approach (50\% of all patients) and 20 patients were operated through the telovelar approach. Patients were examined clinically, radiologically, and functionally using the Karnofsky performance scale at the postoperative period, every 3 months for the first year, and then 6 months thereafter.

Results: Total excision of the tumor was achieved in 27 patients (67.5\% of all patients) and 15 patients (75\% of 20 patients operated via telovelar approach).There were 13 patients (32.5\%) with different postoperative complications, 6 patients underwent telovelar approach, 1 case of superficial infection, 3 cases of bulbar palsy (15\% of patients operated via telovelar approach), and 3 cases of cerebellar mutism (15\% of patients operated via transvermian approach and $7.5 \%$ of all patients).

Conclusion: The telovelar approach can give better exposure to the fourth ventricle in craniocaudal direction and lateral direction without splitting of the vermis. For lesions in the upper half, the ventricletelovelar approach can be used but we have to shift to the transvermian approach for complete resection. The transvermian approach has a higher risk of postoperative cerebellar mutism syndrome particularly in children.
\end{abstract}

Keywords: Fourth ventricle, Approaches, Telovelar, Transvermian

\footnotetext{
* Correspondence: drkhaledneuro@gmail.com

Neurosurgery Department, Ain Shams University Hospitals, Cairo, Egypt
} 


\section{Introduction}

The posterior cranial fossa is the largest and the deepest of the three cranial fossae. Here we found the pathways regulating consciousness, vital autonomic functions, and motor activities and sensory reception for the head, body, and extremities, as well as the center controlling the balance and gait and also the 10 pairs of cranial nerves segmented within the posterior cranial fossa. In addition to that, the posterior cranial fossa is strategically situated at the outlet of the cerebrospinal fluid flow from the ventricular system [1].

Operative approach to the posterior cranial fossa requires an understanding of the relationships of the cerebellum; cranial nerves; brainstem; the cerebellar arteries, veins, and peduncles; and complex fissures between the cerebellum and the brain stem. The relationships of the fourth ventricle to the cerebellar surfaces and the fissures through which the fourth ventricle is approached surgically are among the most complex in the brain [1]

The fourth ventricle is a broad, tent-shaped midline cavity located between the cerebellum and the brain stem. It is connected rostrally through the aqueduct with the third ventricle, caudally through the foramen of magendie with the cisterna magna, laterally through the foramen of the luschka with the cerebellopontine angle. The fourth ventricle has a roof, a floor, and two lateral recesses. It is ventral to the cerebellum, dorsal to the pons and medulla, and medial to the cerebellar peduncles [1].

The area in and around the fourth ventricle may be affected by a variety of neoplasms. Some are unique to the fourth ventricle; others are also found in different locations. Since the fourth ventricle is central in its location in the posterior fossa, it is often affected by neoplasms originating from or traveling to the cerebellum, brain stem, and surrounding meninges and bones [2].

Tumors that occur within the fourth ventricle are ependymoma, subependymoma, choroids plexus papilloma, ganglioglioma, epidermoid, astrocytoma, meningioma, and colloid cyst. Para-fourth ventricular tumors are medulloblastoma, brain stem glioma, cerebellarastrocytoma, hemangioblastoma, metastases, and meningioma [2].

The two most common surgical approaches to the fourth ventricle are the transvermian and the telovelar approaches. Transvermian approach involves incising the inferior vermis of the cerebellum and retracting the two halves of the vermis in the opposite lateral directions. In telovelar approach, the telachoroidea and inferior medullary velum, which form the lower half of the roof of the fourth ventricle, are opened and the lower vermis is retracted as a unit to provide exposure into the fourth ventricle [3].

\section{Telovelar approach}

The medullotonsillar space of the cerebellomedullary fissure and the uvulotonsillar space are sharply dissected to release the tonsils from the uvula and medulla oblongata bilaterally. The two cerebellar tonsils are then retracted laterally to expose the floor of the fissure, i.e., inferior medullary velum and telachoroidea. The telachoroidea which forms the caudal part of the lower half of the roof of the fourth ventricle is incised from the foramen of the magendie and then followed laterally to the foramen of the luschka on both sides [4].

The aim of this study is to compare the results of telovelar and transvermian approaches in the management of fourth ventricular tumors regarding clinical outcome and extent of tumor excision and also to correlate the results of each approach with age of patients, tumor location, size, and pathological nature to evaluate the impact of these factors on each approach.

\section{Patients and methods}

This study includes 40 patients presented at Ain Shams University Hospital and Nasser Institute with the diagnosis of fourth ventriclular tumors in a 4-year period between 2011 and 2015 (Additional file 1). The age of the patients ranges from 5 to 50 years old of both sexes.

All patients were subjected to thorough clinical examination.

All patients investigated by:

1. Routine laboratory investigations (complete blood picture, kidney and liver function tests, and bleeding profile).

2. Radiological investigations: All patients were subjected to computed tomography, and magnetic resonance study of the brain with and without contrast was done for every patient preoperatively.

Magnetic resonance study of the spine with and without contrast was done for patients with ependymoma and medulloblastomato demonstrating spinal subarachnoid dissemination of malignant tumors.

- Cerebral angiography was done for patients with hemangioblastoma when suspected.

- Abdominal computed tomography scan or ultrasound in cases of hemangioblastoma to reveal associated lesions that may be a part of the Von Hipple-Lindau (VHL) disease complex.

3. Histopathological examination was done for all cases to determine the type and grade of the tumor.

\section{Treatment}

Surgical treatment was done in all cases in the form of:

1- Cerebrospinal fluid diversion for hydrocephalic patients through insertion of ventriculoperitoneal 
shunt, external ventricular drain, or endoscopic third ventriculostomy if feasible.

2- Completely randomly choosing an approach, 20 patients were operated through the transvermian approach (50\% of all patients), and 20 patients were operated through the telovelar approach.

\section{Transvermian approach}

- All patients operated in prone position.

- The skin incision is accordingly mid line from just above the union to the spinous process of cervical 2.

- The standard suboccipital craniectomy extend from the inferior edge of the transverse sinus to and including the posterior rim of the foramen magnum which is adherent to the dura and should be cautiously dissected.

- The dura over the midline of the posterior cranial fossa is opened by a Y-shaped incision, the two arms of the $Y$ extending to the transverse sinus and the vertical leg of the $Y$ heading in the cervical direction.

- Splitting the inferior vermis on the sub-occipital surface. The incision extends a variable distance through the uvula, pyramid, tuber, and folium of the vermis, depending on the location and size of the lesion.

- Retracting the two halves of the lower vermis laterally, then opening the telachoroidea and the inferior medullary velum, exposing the full length of the floor from the aqueduct to the obex.

\section{Telovelar approach}

- All patients were operated in prone position.

- The skin incision is accordingly mid line from just above the union to the spinous process of second cervical vertebra.

- The standard suboccipital craniectomy extends from the inferior edge of the transverse sinus to and including the posterior rim of the foramen magnum which is adherent to the dura and should be cautiously dissected.

- The dura over the midline of the posterior cranial fossa is opened by a Y-shaped incision, the two arms of the $Y$ extending to the transverse sinus and the vertical leg of the $Y$ heading in the cervical direction.

- The medullotonsillar space of the cerebellomedullary fissure and the uvulotonsillar space are sharply dissected to release the tonsils from the uvula and the medulla oblongata bilaterally.

- The two cerebellar tonsils are then retracted laterally to expose the floor of the fissure. The telachoroidea is incised from the foramen of the magendie and then followed laterally to the foramen of the luschka on both sides.

3- Total excision was the goal of the surgical treatment of the patients included in the study.

4- Postoperative cerebrospinal fluid shunting was done for some patients when there was clinical and radiological evidence of persistent or developing postoperative hydrocephalus.

5- Radiotherapy was done according to the result of histopathology.

\section{Postoperative follow-up}

1- Patients were examined clinically at the postoperative period of admission, every 3 months at an outpatient clinic in the first year after discharge from hospital, and then every 6 months thereafter.

2- Radiological follow-up of the patients was done in the form of an immediate post-operative computed tomography of the brain with and without contrast and then magnetic resonance image after 3 months and then every 6 months up to 2 years.

3- Outcome of the patients was estimated according to the Karnofsky performance scale.

\section{Results}

\section{Patients' demographics}

Forty patients harboring fourth ventricular tumors underwent resection between 2011 and 2015. There were 25 male $(62.5 \%)$ and 15 female patients (37.5\%), with ages ranging from 5 to 50 years old (mean, $22.5 \pm$ 17 years). $77.5 \%$ of the patients were in the first and second decade when presented.

\section{Histopathology}

This study contained four different types of pathological groups: astrocytoma, medulloblastoma, choroid plexus papilloma, and ependymoma. Fifteen patients harbor ependymoma (37.5\%), 11 patients harbor astrocytoma (27.5\%), 12 patients harbor medulloblastoma (30\%), and two patients harbor choroid plexus papilloma (5\%). Correlating sex and pathological types have been demonstrated in Table 1:

\section{Clinical data}

Thirty patients (75\%) had headache. Thirty-two patients ( $80 \%$ of all patients) presented with vomiting. Nineteen patients (47.5\% of all patients) had suffered from unsteadiness of gait. Eleven patients (27.5\% of all patients) had suffered from diplopia. Fundus examination revealed 23 patients having papilloedema (57.5\% of all patients). 
Table 1 Correlation between sex and pathological types

\begin{tabular}{lllll}
\hline & Male & Female & $x^{2}$ & $P$ value \\
\hline Astrocytoma & 7 & 4 & 3.07 & 0.79 \\
Medulloblastoma & 7 & 5 & & \\
Choroid plexus papilloma & 2 & 0 & & \\
Ependymoma & 9 & 6 & & \\
\hline
\end{tabular}

As regards the cases of astrocytoma, four cases were pilocytic astrocytoma grade I, five patients with astrocytoma were grade II, and two patients were grade III of anaplastic type

Hydrocephalus as diagnosed by computed tomography scanning and magnetic resonance imaging brain was present in 35 patients with hydrocephalic changes (87.5\% of all patients).

Twenty-one patients had cerebellar manifestations (52.5\% of all patients) in the form of uncoordinated movements of the hands, intentional tremors, and gait disturbance.

Ten patients (25\% of all patients) had suffered from sixth nerve palsy. Two patients had seventh nerve palsy (5\% of all patients). The patients' clinical results are summarized in Table 2.

\section{Radiological data}

All patients had both preoperative computed tomography scanning and magnetic resonance imaging of the brain. All patients had postoperative computed tomography brain scanning within the first week postoperative. Patients who had pictures suggestive of medulloblastoma, astrocytoma, or ependymoma were subjected to magnetic resonance imaging for the whole spinal canal searching for seedling. Three patients only ( $7.5 \%$ of all patients) had proven to have seedling in the spinal canal, two patients with ependymoma ( $13.3 \%$ of ependymomas), and one patient with medulloblastoma (8.3\% of medulloblastomas).

Twenty patients were subjected to transvermian approach (50\% of all patients), seven cases of ependymoma

Table 2 Summary of patients' preoperative clinical status

\begin{tabular}{lll}
\hline & No. & Percentage \\
\hline & Symptoms & \\
Headache & 30 & 75 \\
Vomiting & 32 & 80 \\
Unsteadiness & 19 & 47.5 \\
Diplopia & 11 & 27.5 \\
Uncoordinated movement & 21 & 52.5 \\
Signs & & \\
Papilloedema & 23 & 57.5 \\
Hydrocephallus & 35 & 87.5 \\
Six nerve palsy & 10 & 25.0 \\
Seventh nerve palsy & 2 & 5.0 \\
\hline
\end{tabular}

(46.6\% of ependymomas), seven cases of astrocytoma (63.6\% of astrocytomas), five cases of medulloblastoma (41.6\% of medulloblastomas), and one case of choroidplexus papilloma (50\% of choroidplexus papilloma).

Twenty patients (50\% of all patients) were subjected to telovelar approach, eight cases of ependymoma (53.3\% of ependymomas), four cases of astrocytoma (63.6\% of astrocytomas), seven cases of medulloblastoma (58.3\% of medulloblastomas), and one case (50\% of choroidplexus papilloma).

All attempts were done to accomplish total excision of the tumor. The use of surgical microscope and the application of microsurgical techniques had added great advance in the removal of the tumor. This goal was achieved in 27 patients (67.5\% of all patients), 15 (75\%) out of 20 patients operated via telovelar approach, all patients of astrocytoma and choroid plexus papilloma, five (62\%) cases out of eight with ependymoma, five cases $(71.4 \%)$ out of seven with medulloblastoma.

Subtotal removal of the tumor was accomplished in 13 patients (32.5\%), 5 (25\%) of patients operated via telovelar approach and eight (40\%) of patients via transvermian approach. Six cases were ependymoma, four cases were medulloblastoma, and three cases were astrocytoma. In the cases of ependymoma and medulloblastoma, the inferior shell of the tumor was seen to be tethered to the floor and two cases of medulloblastoma were extended laterally into foramen of the luschka. Efforts were done to detach the tumor from the brain stem safely; however, it seemed to be better to leave residual.

Twenty-nine patients were subjected to cerebrospinal fluid diversion operations preoperatively (70\% of all patients). Nine patients out of the 29 patients were operated on emergency bases due to disturbed conscious level. Two patients were operated postoperatively for ventriculoperitoneal shunt within the first $48 \mathrm{~h}$ due to development of clinical and radiological evidence of hydrocephalus.

As regards postoperative complications, there were 13 patients with different postoperative complications (32.5\% of all patients). Six patients underwent telovelar approach $(30 \%$ of patients operated via telovelar approach and $15 \%$ of all patients), one case of superficial infection (5\% of patients operated via telovelar approach and $2.5 \%$ of all patients), three cases of bulbar palsy (15\% of patients operated via telovelar approach and $7.5 \%$ of all patients), one patient with ependymoma out of eight $(12.5 \%$ of ependymomas operated via telovelar approach and $6.6 \%$ of all ependymomas), and two patient with medulloblastoma $(28.5 \%$ of medulloblastomas operated via telovelar approach and $16.6 \%$ of all medulloblastomas).

There were three cases of cerebellar mutism (15\% of patients operated via transvermian approach and $7.5 \%$ of all patients), one patient with ependymoma out of seven 
(14.2\% of ependymomas operated via transvermian approach and $6.6 \%$ of all ependymomas), one patient with medulloblastoma ( $20 \%$ of medulloblastomas operated via transvermian approach and $8.3 \%$ of all medulloblastomas), and one patient with astrocytoma (14.28\% of astrocytomas operated via transvermian approach and $9 \%$ of all astrocytomas).

These three cases were operated via transvermian approach with small vermian incision not extended much upward. They were children less than 10 years with delayed onset of mutism, emotional liability, unimpaired consciousness, and no disturbances of organs of phonation, being able to talk in the first few hours after surgery. Follow-up computed tomography scanning and magnetic resonance imaging of these patients showed no obvious abnormality of infarction, hemorrhage, or injury to a vital neurosurgical structure. The only positive data in radiological picture was mild to moderate local edema. Two cases have improved with transient course over 3 weeks with no residual deficits while the third case who was the youngest (6 years) with large-sized tumor has extended course with partial improvement over 4 months.

There were 26 patients (65\% of all patients) who had received postoperative radiotherapy, of them there were nine patients with ependymoma (22.5\% of all patients, $60 \%$ of ependymomas), 12 patients with medulloblastoma (30\% of all patients, $100 \%$ of medulloblastomas), seven patients with astrocytoma (17.5\% of all patients, $63.6 \%$ of astrocytomas); five patients with astrocytoma were grade II and two patients were grade III anaplastic type, and three of these patients had whole neuroaxis radiotherapy two of them was ependymoma (5\% of all patients, $13.3 \%$ of ependymomas) and the third patient was medulloblastoma $(2.5 \%$ of all patients, $8.3 \%$ of medulloblastomas).

The final prognosis of these patients was assessed by comparing clinical manifestation of each group preoperative and postoperative using the Karnofsky performance status scale (Table 3 ).

\section{Classified into three groups}

The first group contains patients with improvement of the Karnofsky performance status scale, 20 patients $(50 \%$ of all patients) improved one degree from $(60 \%-70 \%)$ to $(70 \%-80 \%)$ of the Karnofsky performance status scale.

Table 3 Postoperative functional status according to (Karnofsky performance scale)

\begin{tabular}{lll}
\hline & No. & Percentage \\
\hline 1st group & 20 & 50 \\
2nd group & 10 & 25 \\
3rd group & 10 & 25 \\
\hline
\end{tabular}

The second group contains patients with the same Karnofsky performance status scale, ten patients (25\% of all patients) ranged from 60 to $90 \%$ of the Karnofsky performance status scale.

The third group contains patients with deterioration of the Karnofsky performance status scale, ten patients (25\% of all patients) ranged from one to two degrees of deterioration of the Karnofsky performance status scale, the least scale was $40 \%$.

\section{Discussion}

This study includes 40 patients presented at Ain Shams University Hospital and Nasser Institute with the diagnosis of fourth ventricular tumors in a 4-year period between 2011 and 2015. The age of the patients ranges from 5 to 50 years old of both sexes. There were four different pathological types, namely fourth ventricularependymoma, choroid plexus tumors, medulloblastoma, and cerebellarastrocytoma.

This study showed that the incidence in cases of ependymoma was $37.5 \%$ (15 patients) of all patients of fourth ventricular tumors, in comparison to $25 \%$ in the work of El-Bahy on the (telovelar approach to fourth ventricle) [4].

The male to female ratio in case of ependymoma was 1.5:1; these results are more or less compatible with the results reported by Grill et al. in their study that shows approximately equal in males and females [5].

The incidence of astrocytoma was 27.5\% (11 patients) of all patients compared to $33 \%$ of all tumors as reported by Akay in his work on those tumor [6] and 19\% in the work of El-Bahy [4].

The incidence of medulloblastoma was 30\% (12 patients) of all fourth ventricular tumors included in this study which is higher than percentage reported by Smoll and Drummond as 20\% [7] and higher than 19\% as reported by El-Bahy [4].

In this thesis, we had noticed that the male to female ratio in cases of medulloblastoma was 1.4:1, while according to the work of Polkinghorn and Tarbell, the male to female ratio was 2:1 with male predominance [8].

As regards choroid plexus tumors, we found the incidence was $5 \%$ (two patients) of all fourth ventricular tumors, which are more compatible with $6.25 \%$ as reported by El-Bahy [4].

The two cases of choroid plexus tumors are two males, while it was2.8:1 in the work of Bauchet and his colleagues [9].

In cases of astrocytoma, the mean age at diagnosis was 27 years, which is a pit higher than 18 years in the work of Riva and Giorgi [10].

While in cases of medulloblastoma in this study, the mean age at diagnosis was 7.9 years, which is compatible with the study of Smoll and Drummond that shows the peak incidence is between 5 and 9 years of age. 


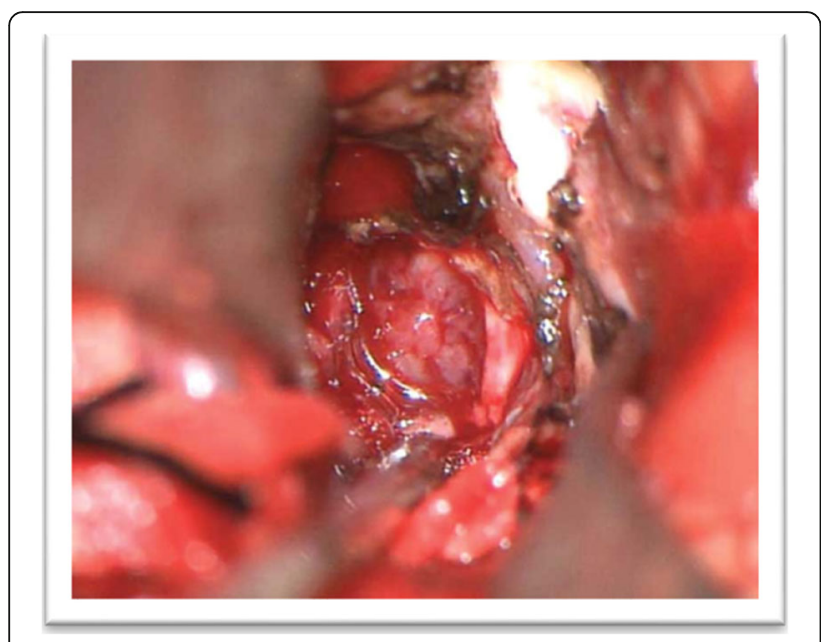

Fig. 1 The dura over the midline of the posterior cranial fossa is opened by a $Y$-shaped incision, the two arms of the $Y$ extending to the transverse sinus and the vertical leg of the $Y$ heading in the cervical direction

Approximately $70 \%$ of patients are diagnosed before the age of 20. There is a slight increase in incidence between the ages of 20 and 24 years, and the disease is rare after the fourth decade, consistent with its embryonal origin [7].

Radiologically, the presence of hydrocephalus in cases of patients suffering from fourth ventricular tumors is a hallmark dragging attention to patients suffering from space occupying lesion obstructing the cerebrospinal fluid pathways at the level of the fourth ventricle with a subsequent famous triad of increased intracranial pressure composed of headache especially at the morning, nausea and vomiting, and blurring of vision. In case of ependymoma, the incidence of hydrocephalus was $100 \%$, which is compatible with $100 \%$ in the work of Amirian and his colleagues [11]. In this study, this picture was present in $91.6 \%$ of patients with medulloblastoma (11 patients out of 12). According to the work of Anthony and his colleagues, hydrocephalus occurs in a percentage of $94 \%$ in cases on medulloblastoma [12].

In this study, 31 patients ( $80 \%$ of all patients) had preoperative cerebrospinal fluid diversion, two patients were operated postoperatively for ventriculoperitoneal shunt within the first $48 \mathrm{~h}$ due to development of clinical and radiological evidence of hydrocephalus, those patients were one patient with ependymoma and one patient with astrocytoma, and according to the work of Anthony and his colleagues, the percentage of preoperative shunting was $86 \%$ [12].

The aim of the surgery was gross total removal of the tumor with minimal trauma to the surrounding neural and vascular structures. We utilized standard microsurgical techniques. In this study, all patients were operated upon in a prone position as performed by Noman and Martin, the occipital bone and posterior arch of cervical 1 were exposed. A small midline suboccipital craniotomy was cut, exposing only the cerebellar tonsils and inferior vermis. The posterior arch of the cervical 1 was removed if the tonsils were seen descended to this level or below the preoperative imaging.

In our series, the suboccipital craniectomy was extended to the foramen magnum in all cases (100\%), The upper cervical spine (cervical 1 and cervical 2) was removed in eight cases (20\% of cases), four cases (26.6\%) of ependymoma out of 15 , two cases (16.6\%) of medulloblastoma out of 12 , and two cases (18\%) astrocytoma out of 11. In our series, this was done in cases where the tonsils were seen descend to this level or below on the preoperative imaging as a standard indication to remove cervical 1 arch. Deshmukh et al. compared the telovelar approach with and without cervical 1 posterior arch removal to the transvermian approach. They found that additional removal of the cervical 1 arch offered a larger
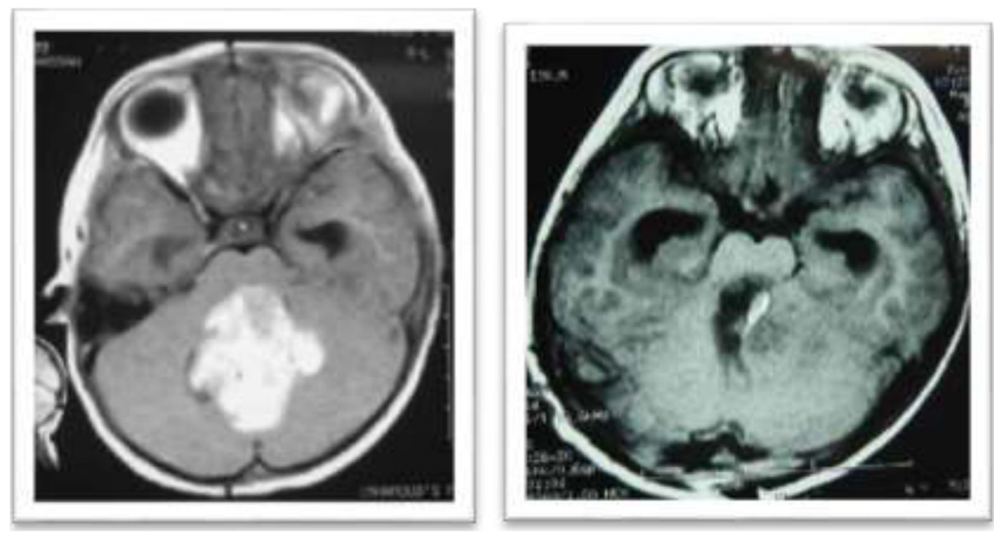

Fig. 2 (Left) Pre-op MRI brain axial cut show fourth ventricular mass with homogenous enhancement and hydrocephalus operated via the transvermian approach. (Right) Immediate postoperative MRI brain axial view with contrast shows no residual, no hematoma 


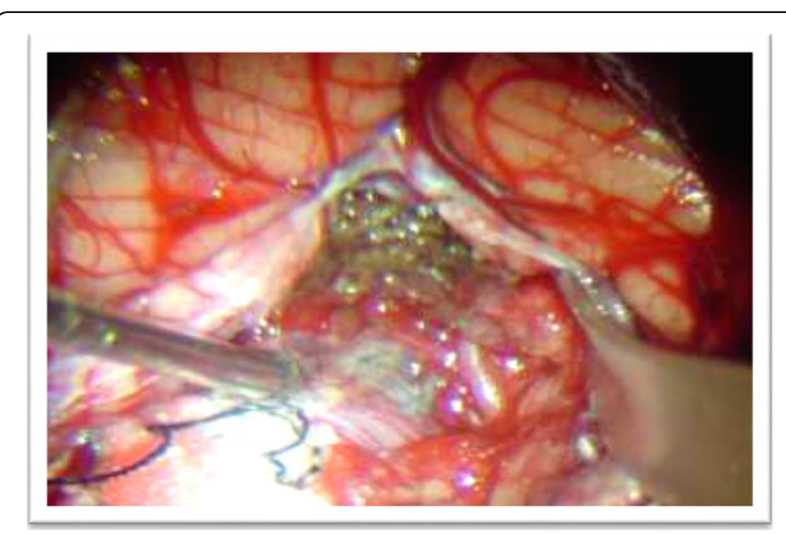

Fig. 3 Exposure of the fourth ventricle under microscope; the two cerebellar tonsils are retracted laterally to expose the floor of the fissure, i.e., inferior medullary velum and telachoroidea. The telachoroidea which forms the caudal part of the lower half of the roof of the fourth ventricle is incised from the foramen of the magendie and then followed laterally to the foramen of the luschka on both sides

working area than the transvermian approach, obviating the sole advocated advantage of the transvermian approach to the rostral half of the fourth ventricle. Nevertheless, we found this procedure necessary only if the tonsils were pushed below the level of the foramen magnum by the tumor. Gentle manual intermittent retraction could allow for a satisfactory perspective of the upper fourth ventricle.

In this study, 20 patients were operated via transvermian approach, and the other 20 patients were operated via telovelar approach.

The 20 patients were subjected to transvermian approach (50\% of all patients), seven cases of ependymoma (46.6\% of ependymomas), seven cases of astrocytoma (63.6\% of astrocytomas), five cases of medulloblastoma (41.6\% of medulloblastomas), and one case of choroid plexus papilloma (50\% of choroid plexus papillomas).

In the splitting of the inferior vermis of the suboccipital surface, the extent of the incision through the inferior vermis has been poorly described. The incision extends a variable distance through the uvula, pyramid, tuber, and folium of the vermis, depending on the location and size of the lesion. The vermian incision was exposing the underlying nodule, which was necessarily incised along with the telachoroidea and the inferior medullary velum to gain access to the fourth ventricle [13].

Retraction of the two halves of the lower vermis laterally provide approximately 1 to $2 \mathrm{~cm}$ of working space between the two edges of the inferior vermis. After the telachoroidea and the inferior medullary velum had been opened through a mid-line incision, the full length of the floor (mean length $4 \mathrm{~cm}$ ) from the aqueduct to the apex could be exposed. Laterally, the limit of exposure was maximal at the level of the striaemedullaris [3].

Total excision was accomplished in 12 patients via the transvermian approach (60\% of patients operated via transvermian approach), four (57.1\%) out of seven patients with ependymoma, three $(60 \%)$ cases out of five with medulloblastoma, four (57.1\%) cases out of seven with astrocytoma, and the one patient with choroid plexus papilloma. Total excision accomplished in 15 (75\%) out of twenty patients of whom were operated via the telovelar approach is compatible with the $70 \%$ in the work of Noman and Martin, all patients of astrocytoma and choroid plexus papilloma, five (62.5\%) cases out of eight with ependymoma, and five $(71.4 \%)$ cases out of seven with medulloblastoma.

Subtotal removal of the tumor was accomplished in eight patients via transvermian approach (40\% of patients operated via transvermian approach), three $(42.8 \%)$ out of seven patients with ependymoma, and two cases (40\%) out of five with medulloblastoma, three (42.8\%) cases out of seven with astrocytoma.

The 20 patients (50\% of all patients) were subjected to telovelar approach, eight cases of ependymoma $(53.3 \%$ of ependymomas), four cases of astrocytoma (63.6\% of astrocytomas), seven cases of medulloblastoma (58.3\% of
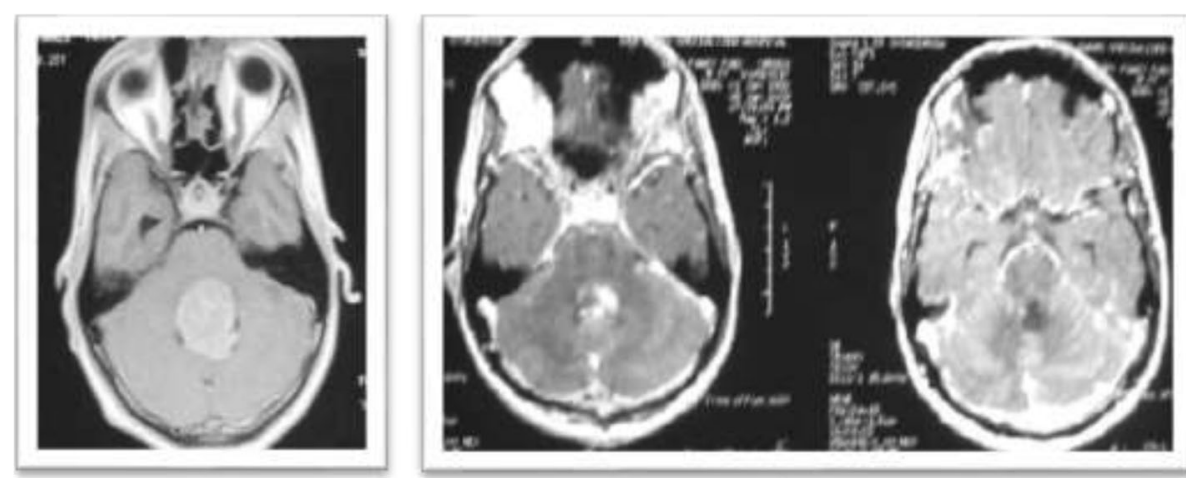

Fig. 4 (Left) Pre-op MRI brain axial cuts with contrast shows fourth ventricular mass (fourth ventricular choroid plexus papilloma) with homogenous enhancement operated via transvermian approach. (Right) Near total resection was achieved through telovelar approach with small residual on the fourth ventricular floor. Results 


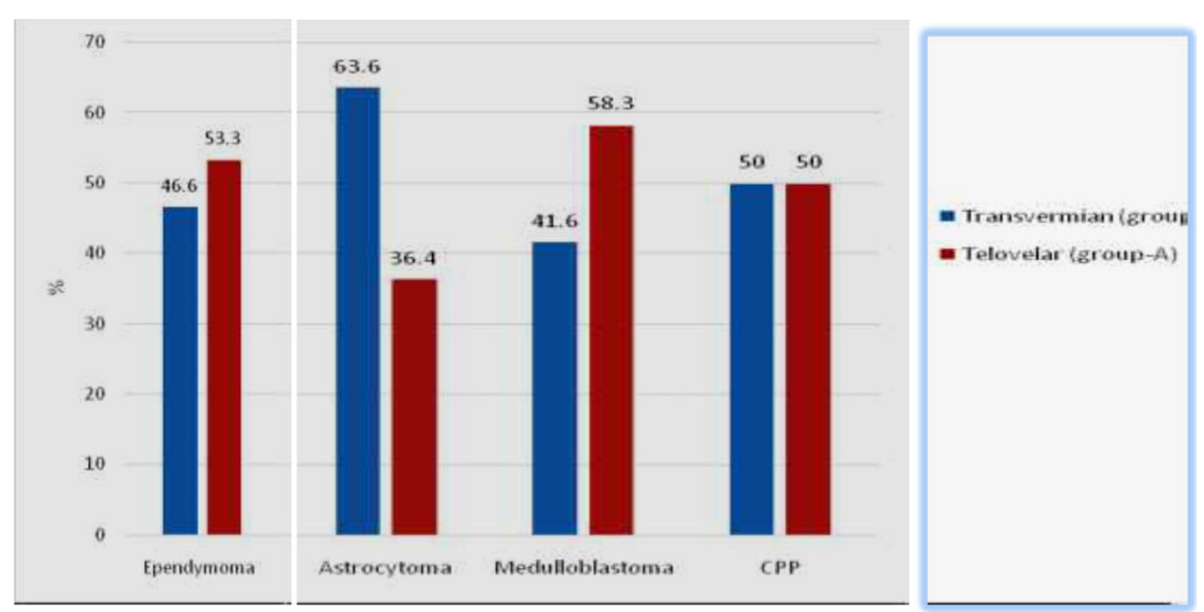

Fig. 5 Types of tumors and operations done

medulloblastomas), and one case of choroid plexus papilloma (50\% of choroid plexus papillomas).

Under the operating microscope, the cerebellar tonsil on the side of the greatest tumor bulk was gently elevated to reveal the foramen of the magendie, the telachoroidea, and the inferior medullary velum. The inferior aspect of a large tumor was often evident protruding through the foramen of the magendie, with the telachoroidea and inferior medullary velum stretched thinly over its surface.

Total excision accomplished in 15 (75\%) out of 20 patients of whom were operated via telovelar approach was compatible with the $70 \%$ in the work of Noman and Martin [14].

All patients of astrocytoma and choroid plexus papilloma, five (62.5\%) cases out of eight with ependymoma, and five (71.4\%) cases out of seven with medulloblastoma. The experience of El-Bahy with large fourth ventricular tumors, with an extension to the aqueduct above, obex below, and lateral recesses bilaterally, indicates that telovelar approach alone is enough, and vermian incision was not needed. Opening of thinned inferior medullary velum and telachoroidea on both sides with multidirectional uvular retraction was found to be sufficient to deal with large tumors [4].

The telovelar approach provides greater horizontal angle of exposure at the level of the foramen of the luschka than the transvermian. There is no significant difference in the vertical angle to the roof of the fourth ventricle. Removal of the cervical 1 posterior arch significantly augments the vertical angle of the telovelar at both caudal and rostral levels of the forth ventricle. The telovelar with the cervical 1 posterior arch removal offers the greatest working area when addressing lesions of the fourth ventricle [15].

This result is due to the good exposure of the fourth ventricle through the telovelar approach through three steps of exposure according to the location and size of the lesion without neural tissue injury, and these three steps are:

1- Extensive (aqueduct) opening method

2- Lateral wall opening method

3- Lateral recess opening method [16]

Telovelar approach is better in the exposure of the lesions in the fourth ventricle that extend upward to the aqueduct by using the extensive aqueductal opening method in which the uvulotonsillar and medullotonsillar spaces are dissected bilaterally without vermian incision, which was at least $1 \mathrm{~cm}$ in the transvermian approach [16].

Postoperative computed tomography scan was done in 40 cases with and without contrast administration within $48 \mathrm{~h}$ after surgery to detect the presence of postoperative hydrocephalus in comparison to the degree of hydrocephalus in preoperative images and to evaluate the extent of tumor removal [17]. The extent of resection should be assessed with the preoperative and early postoperative imaging studies; this avoids the surgeon's bias, which often over- or underestimates the true amount of tumor removed.

In this study, patients who were histologically proven to be having pendymoma or medulloblastoma were investigated for spinal metastasis after 2 weeks postoperative. Three patients only (7.5\% of all patients) had been proven to have seedling in the spinal canal and two patients with ependymoma (13.3\% of ependymomas), which is higher than the $10 \%$ in the work of Ligia and his colleagues [18].

As regards postoperative complications, there were 13 patients with different post-operative complications (28\% of all patients). Six patients underwent telovelar approach $(30 \%$ of patients operated via telovelar approach) the same as that in the work of Noman and Martin [14]. 
There were three cases of bulbar palsy (15\% of patients operated via telovelar approach and $7.5 \%$ of all patients), one patient with ependymoma out of eight (12.5\% of ependymomas operated via telovelar approach) compared to $25 \%$ in the work of El-Bahy and two patients with medulloblastoma ( $28.5 \%$ of medulloblastomas operated via telovelar approach).One case of cerebrospinal fluid leak (5\% of patients operated via telovelar approach and $2.5 \%$ of all patients) with astrocytoma (25\% of astrocytomas operated via telovelar approach and $9 \%$ of all astrocytomas) compared to $10 \%$ in the work of Noman and Martin [14].

In this study, no cases developed cerebellar mutism in patients who underwent telovelar approach which is the same as that in the work of El-Bahy. Seven patients underwent transvermian approach (35\% of patients operated via transvermian approach and $17.5 \%$ of all patients).

There were three cases of bulbar palsy ( $15 \%$ of patients operated via transvermian approach and $7.5 \%$ of all patients), one patient with ependymoma out of seven (14.2\% of ependymomas operated via the transvermian approach and $6.6 \%$ of all ependymomas), one patient with medulloblastoma ( $20 \%$ of medulloblastomas operated via the transvermian approach and $8.3 \%$ of all medulloblastomas), and one patient with astrocytoma (14.28\% of astrocytomas operated via the transvermian approach and $9 \%$ of all astrocytomas).

Three cases of cerebellar mutism (15\% of patients operated via the transvermian approach and $7.5 \%$ of all patients) is compatible with the incidence of posterior fossa syndrome as reported by Pollack and his colleagues was $8.5-13 \%$. One patient with ependymoma out of seven $(14.2 \%$ of ependymomas operated via transvermian approach and $6.6 \%$ of all ependymomas), one patient with medulloblastoma ( $20 \%$ of medulloblastomas operated via transvermian approach and $8.3 \%$ of all medulloblastomas), and one patient with astrocytoma (14.28\% of astrocytomas operated via transvermian approach and $9 \%$ of all astrocytomas).

In our study, the posterior fossa syndrome occurs in cases operated via transvermian approach with small vermian incision not extended much upward. This is compatible with the work of Riva and Giorgi that suggest that cerebellar mutism is secondary to edema in the deep cerebellar nuclei and may be related to the manipulation of the vermis [10].

As regards the spinal metastasis in cases of medulloblastoma, it was one patient with medulloblastoma ( $8.3 \%$ of medulloblastomas), which is lower than the work of Jonathan and Cohen that shows a very wide range of spinal dissemination $13-43 \%$, and they show also that dissemination at diagnosis is frequent in patients younger than 5 years old [19].
Although prophylactic whole craniospinal irradiation was advocated at one time for all resected ependymomas, local recurrence is the predominant site of failure and whole spine irradiation does not appear to improve survival. More extensive radiation fields are indicated if there is evidence of tumor dissemination based upon neuroimaging or cerebrospinal fluid cytology [20].

The rationale for additional radiation to the tumor bed is based upon the observation that 50 to $70 \%$ of recurrences occur in the posterior fossa. Although some investigators include the entire posterior fossa in the boost volume, current protocols are investigating the use of reduced posterior fossa high-dose volume boost in order to spare normal brain from excess radiation exposure [21].

As regards the cases of astrocytoma, postoperative radiotherapy had been received in seven patients with astrocytoma (63.6\% of astrocytomas), five patients with astrocytoma were grade II and two patients were grade III anaplastic type which is compatible with the work of Conway and his colleagues, who reported that astrocytomas cannot be totally resected and even those histologically of low grade may recur without radiotherapy.

It is important to emphasize that the most important prognostic indices for satisfactory results in case of fourth ventricular tumors depends greatly on early diagnosis of the patient. Provided that there is no tumor invasion into the brain stem, cerebellopontine angles or supratentorial as seen by computed tomography scanning or magnetic resonance imaging of the brain, it is mandatory to choose the approach and degree of removal as the pathological nature of the lesion and vital neural tissue infiltration which are the limiting factors for total tumor removal. So, near total removal is acceptable in cases where complete removal may endanger function or life.

\section{Conclusion}

The most common fourth ventricular tumors in this study were ependymoma, astrocytoma, and medulloblastoma, which are more common in the child age group. Choroid plexus papilloma usually affects the fourth ventricle in the adult age group.

The main presentation of fourth ventricular tumors is the symptoms and signs of increased intracranial pressure due to obstruction of the CSF pathway, and the main treatment is the tumor excision (Figs. 1, 2, 3,4 , and 5).

Ventriculoperitoneal shunt is needed if total tumor excision is not available due to infiltration of the brain stem or cerebellar peduncle and also in cases presented with severe obstructive hydrocephalus threatening their lives. 


\section{There are two approaches to the fourth ventricular tumors \\ Telovelar approach}

Telovelar approach can give better exposure to the fourth ventricle in the craniocaudal direction and lateral direction without splitting of the vermis or causing injury to vital neural structures, so this can be used in lesions in the lower half of the fourth ventricle, lesions occupying the whole ventricle, and lesions extending into foramina of the luschka and magendi, while in lesions in the upper half, we can begin with the telovelar but if it is impossible to excise the tumor totally we have to turn to transvermian approach.

\section{Transvermian approach}

Transvermian approach may be complicated by postoperative cerebellar mutism syndrome particularly in children. The most affecting factors in the prognosis of fourth ventricular tumors are the pathological nature of the lesions, the degree of tumor excision, and the infiltration of vital neural structure. Total removal of tumors focally attached to critical areas in the fourth ventricle should not be attempted at the expense of the patient's morbidity and mortality. Near total excision is acceptable in cases where complete removal may endanger function or life.

\section{Radiotherapy}

Radiotherapy to either the primary site or whole neuroaxis radiation improves the prognosis in cases of medulloblastoma, ependymoma, and astrocytoma.

\section{Additional file}

Additional file 1: Arabic informed consent. (DOCX 129 kb)

\section{Acknowledgements}

The authors would like to thank and appreciate prof. Khaled El Bahy for his valuable contribution into material of this study.

\section{Funding}

None.

\section{Availability of data and materials}

Can be shared upon personal justified request to 2nd author.

\section{Study design}

This study is a retrospective clinical study.

\section{Authors' contributions}

AFT is the main author, performed most of the surgeries and approaches in this study, did the follow-ups of the cases, and reviewed the data. KSE is the corresponding author, performed some of the surgeries, and did the final editing, discussion, and review of statistics. Both authors read and approved the final manuscript.

\section{Ethics approval and consent to participate}

The study was approved by Ain Shams University Faculty of Medicine Ethical Committee. FMASU R 71/ 2018. Informed consent to participate was given by all patients or parents (Additional file 1).
Consent for publication

(Non applicable). Retrograde study. Personal information's were removed.

Competing interests

The authors declare that they have no competing interests.

\section{Publisher's Note}

Springer Nature remains neutral with regard to jurisdictional claims in published maps and institutional affiliations.

Received: 8 August 2018 Accepted: 4 February 2019

Published online: 14 February 2019

\section{References}

1. Rhoton LA. The Posterior Cranial Fossa: Microsurgical Anatomy and Surgical Approaches. Neurosurgery. 2000;47(3):S5-S6. https://doi.org/10.1097/ 00006123-200009001-00005.

2. Cohen AR. Surgical disorders of the fourth ventricle. Cambridge: Blackwell science; 1996. p. 147-60.

3. Necmettin T, Arthur J, Rhoton AL, Yasuda A. Comparison of the transvermian and telovelar approaches to the fourth ventricle. J Neurosurg. 2004:101:484-98.

4. El-Bahy K. Telovelar approach to the fourth ventricle: operative findings and results in 16 cases. Acta Neurochir (Wien). 2005;147:137-42

5. Grill J, Pascal C, Chantal K. Childhood ependymoma: a systematic review of treatment options and strategies. Paediatr Drugs. 2003:5:533.

6. Akay KM, Izci Y, Baysefer A, et al. Surgical outcomes of cerebellar tumors in children. PediatrNeurosurg. 2004;40(5):220-5.

7. Smoll NR, Drummond KJ. The incidence of medulloblastomas and primitive neurectodermaltumours in adults and children. J Clin Neurosci. 2012;19:1541.

8. Polkinghorn WR, Tarbell NJ. Medulloblastoma: tumorigenesis, current clinical paradigm, and efforts to improve risk stratification. Nat Clin Pract Oncol. 2007:4(5):295-304

9. Bauchet L, Rigau V, Mathieu-Daudé H, Fabbro-Peray P, Palenzuela G, Figarella-Branger D, et al. Clinical epidemiology for childhood primary central nervous system tumors. J Neurooncol. 2009;92(1):87-98.

10. Riva D, Giorgi C. The cerebellum contributes to higher functions during development. Evidence from a series of children surgically treated from posterior fossa tumors. Brain. 2000;123:1051-61.

11. Amirian ES, Armstrong TS, Aldape KD, et al. Predictors of survival among pediatric and adult ependymoma cases: a study using surveillance, epidemiology, and end results data from 1973 to 2007. Neuroepidemiology. 2012;39:116

12. Raimondi AJ, Tomita T. Hydrocephalus and infratentorial tumors. Incidence, clinical picture, and treatment. J Neurosurg. 1981;55(2):174-82.

13. Ziyal IM, Sekhar LN, Salas E. Subtonsillo-transcerebellomedullary approach to lesions involving the fourth ventricle, the cerebellomedullary fissure and the lateral brain. Br J Neurosurg. 1999:1:276-84.

14. Zaheer SN, Wood M. Experiences with the telovelar approach to fourth ventricular tumors in children. Pediatr Neurosurg. 2010:46:340-3.

15. Figueiredo EG, Deshmukh V, Deshmukh P, Crawford NC, Preul MC, Spetzler RF. The telovelar approach to the fourth ventricle: an anatomical comparison, American Association of Neurological Surgeons Abstract; 2009. p. Article ID: 59130.

16. Matsushima T, Inoue T, Inomura T, et al. Transcerebellomedullary fissure approach with special reference to methods of dissecting the fissure. J Neurosurg. 2001;94:257-64.

17. Chen $C Y$, Zimmerman RA. Imaging of brain tumors in infants and children. In: Tindall GT, et al., editors. The practice of neurosurgery. 1st ed. Philadelphia: Williams \& Wilkins; 1997. p. 755-78.

18. Macedo LT, Rogerio F, Pereira EB, de Souza Queiroz L, Carvalheira JBC. Cerebrospinal tumor dissemination in a patient with myxopapillary ependymoma 2011 by American Society of Clinical Oncology; 2011.

19. Miller JP, Cohen AR. Surgical management of tumors of the fourth ventricle. In: Schmidek HH, Sweet WH, editors. Operative neurosurgical techniques, vol. 61. 6th ed. Philadelphia: Saunders, Elsevier; 2006. p. 881-913.

20. Merchant TE, Fouladi M. Ependymoma: new therapeutic approaches including radiation and chemotherapy. J Neuro-Oncol. 2005:75:287.

21. Wolden SL, Dunkel IJ, Souweidane MM, et al. Patterns of failure using a conformal radiation therapy tumor bed boost for medulloblastoma. J Clin Oncol. 2003;21:3079. 